\title{
Novel Eudragit'-based polymeric nanoparticles for sustained release of simvastatin
}

\author{
Deborah Fernandes Rodrigues ${ }^{\circledR 1}$, Renê Oliveira do Couto', \\ Rubén Dario Sinisterra², Carlos Eduardo de Matos Jensen ${ }^{1^{*}}$ \\ ${ }^{1}$ Federal University of São João del-Rei, Midwest Campus Dona Lindu, Divinópolis, Minas Gerais, Brazil, \\ ${ }^{2}$ Department of Chemistry, Federal University of Minas Gerais, Belo Horizonte, Minas Gerais, Brazil
}

\begin{abstract}
This paper reports on the development of nanoparticles aiming at the in vitro controlled release of simvastatin (SVT). The nanoparticles were prepared by the nanoprecipitation method with polymers Eudragit ${ }^{\circledR}$ FS30D (EDGFS) or Eudragit ${ }^{\circledR}$ NE30D (EDGNE). EDGFS+SVT nanoparticles showed mean size of $148.8 \mathrm{~nm}$ and entrapment efficiency of 76.4\%, whereas EDGNE+SVT nanoparticles showed mean size of $105.0 \mathrm{~nm}$ and entrapment efficiency of $103.2 \%$. Infrared absorption spectra demonstrated that SVT incorporated into the polymer matrix, especially EDGNE. Similarly, the differential scanning calorimeter (DSC) curve presented no endothermic peak of fusion, indicating that the system is amorphous and the drug is not in the crystalline state. The maintenance of SVT in the amorphous state may favors its solubilization in the target release sites. In the in vitro dissolution assay, the SVT incorporated into the EDGFS+SVT nanoparticles showed a rapid initial release, which may be related to the $\mathrm{pH}$ of the dissolution medium used. Regarding the EDGNE+SVT nanoparticles, the in vitro release occurred in a bimodal behavior, i.e., an initial "burst" followed by a sustained delivery, with the kinetics of drug release following Baker-Lonsdale's mathematical model. All these features suggest the nanoparticulate system's potential to modulate SVT delivery and enhance its bioavailability.
\end{abstract}

Keywords: Simvastatin. Nanoparticles. Poly-methylmethacrylate. Dissolution. Drug delivery systems.

\section{INTRODUCTION}

Simvastatin (SVT) (Figure 1) is a drug that belongs to a class of substances called statins, commonly used to reduce the production of cholesterol. It acts by suppressing the endogenous cholesterol biosynthesis as a function of the competitive inhibition of 3-hydroxy3-methylglutaryl coenzyme-A (HMG-CoA) reductase - an enzyme that catalyzes the initial and rate-limiting step of the production of cholesterol. This inhibition contributes to the control of hypercholesterolemia, which is one of the risk factors for the development of atherosclerosis (Stone et al., 2014; Da Cruz et al., 2017).

*Correspondence: C. E. M. Jensen, Campus Centro-Oeste Dona Lindu, Universidade Federal de São João del-Rei, R. Sebastião Gonçalves Coelho, 400, CEP 35501-296, Chanadour - Divinópolis, MG. Tel: (37) 36904497. E-mail: jensen@ufsj.edu.br
In conventional therapy, SVT have been orally administered by mean of polymer coated tablets in daily doses ranging from $10 \mathrm{up}$ to $80 \mathrm{mg}$. SVT has low solubility and high permeability $(65-85 \%)$ in the gastrointestinal tract (GIT). Owing to these characteristics, SVT is included in Class II of the Biopharmaceutical Classification System (BCS) guidance. In Class II, drug dissolution in the GIT is considered the rate-limiting step for oral bioavailability (Fattahi et al., 2016).

The SVT is a lipophilic natural statin, administered in its lactone form which has to be metabolized in the liver to become available for binding with HMG-CoA reductase inside hepatocytes and thus act as lipidlowering drug. For that, after being released from a formulation in the intestine or any other delivery route, SVT is transported thorough the plasma membrane by passive diffusion to inside the liver, where is 
metabolized by the cytochrome P450 family of enzymes, more specifically by CYP3A4 (Cunha e Rocha, Pereira, Rodrigues, 2018).

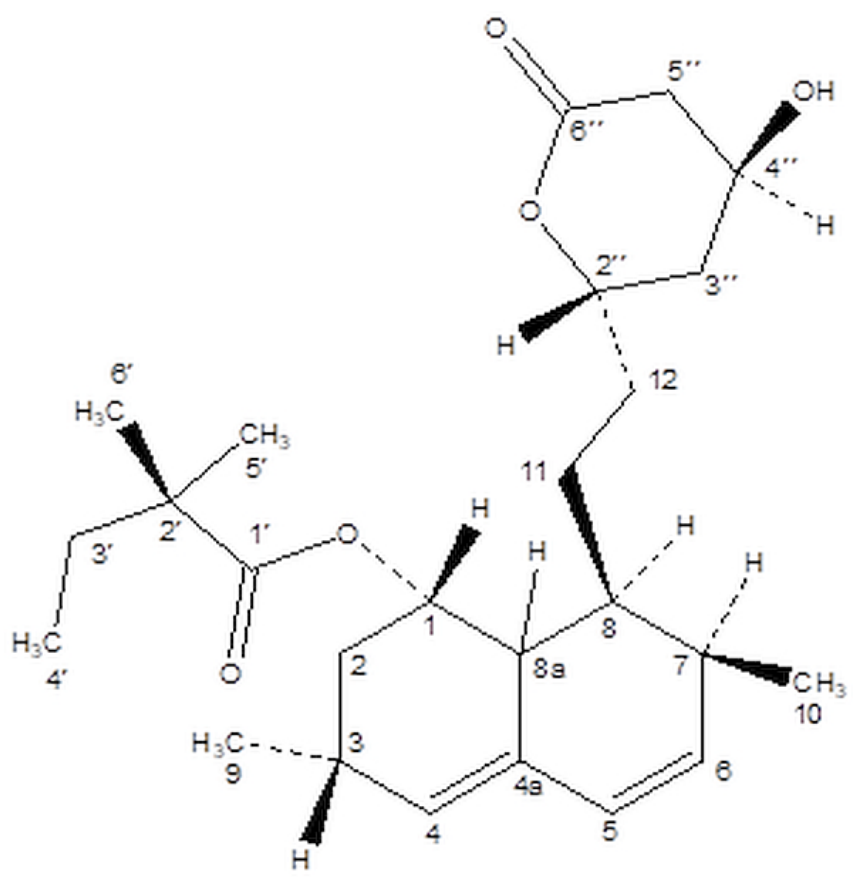

FIGURE 1 - Molecular structure of simvastatin (SVT) or 8 - [2 - (4-hydroxy-6-oxo-oxan-2-yl) ethyl] - 3, 7-dimethyl-1, 2, 3, 7, $8,8 \mathrm{a}$ - hexa hydronaphthalene-1-yl] -2, 2 - dimethylbutanoate (IUPAC) [(1S,3R,7S,8S,8aR)-8-[2-[(2R,4R)-4-hydroxy6 - ox o ox an-2-yl] ethyl]-3,7-dimethyl-1,2,3,7,8,8 ahexahydronaphthalen-1-yl] 2,2-dimethylbutanoate.

Nighttime administration of SVT is required due to its short half-life $(1.9-3 \mathrm{~h})$ and the steroid synthesis is more active during the night (Taha, 2016). Such drug also possess low bioavailability of SVT $(<5 \%)$ and, to achieve the desired effects, a higher dose can be administered, significantly increasing the possibility of adverse and/ or toxic effects, which may be directly associated with reduced adherence to treatment (Thompson et al., 2016).

Typically, the low bioavailability of SVT has been attributed to: i) first-pass metabolism by the liver; ii) clearance within the gastrointestinal tract; iii) limited cell permeability; and iv) limited aqueous solubility. Although, paradoxically, the great first-pass uptake of the drug (78-97\%) contributes to its low bioavailability, it drives the LDL-C lowering properties since the liver is the main site of endogenous cholesterol synthesis (Korani et al., 2018). Thereby, developing a stable delivery system for SVT with enhanced bioavailability following administration displays a complex task and a great challenge for pharmaceutical scientists.

As long as the hepatic cholesterologenesis is a key point of SVT action, a liver-targeted delivery system which enable enhanced uptake into hepatocytes would be of great interest for clinical practice. In this pursuit, an ideal SVT delivery system should be able to enhance hepatic bioavailability by an efficient drug transport through the intestinal and mucosal barriers and an optimized intrahepatic release of such drug (Petyaev, 2015).

By meeting these basic requirements, polymeric nanoparticles and other nanometric delivery systems which enable a targeted and controlled release of statins have been considered a step forward in the treatment hypercholesterolemia as compared to the conventional dosage forms (Shilpi et al., 2017; Alshora et al., 2018). This may be explained in basis of their capability to: i) diminish fluctuations in the drug plasma concentration; ii) provide an enhanced hepatic bioavailability of such drugs by increasing their uptake by absorptive endocytosis; and iii) lowering exposure of peripheral tissues to the statins (Petyaev, 2015). Besides the improvement in the drug effects, the beneficial aspects achieved by nanotechnology would include decreased dosing, minimized side-effects and raised patient compliance and adhesion to the treatment (Korani et al., 2018).

Polymers used in nanoparticle preparation can be biodegradable or non-biodegradable. In the human body, biodegradable polymers are known to undergo random hydrolysis of covalent bonds, especially ester bonds, thereby reducing the molar mass of the polymer. The reduced molar mass causes the nanoparticulate system to collapses and undergo bioerosion, with consequent loss of its physical integrity. The resulting fragments have greater solubility, are not toxic, and are metabolized and eliminated naturally by the organism. Examples of these polymers include polyesters from lactic acid and from glycolic acid (Basu et al., 2016).

Non-biodegradable polymers, in turn, can be exemplified by polyacrylates and polymethylmethacrylates (commercially known as Eudragit $^{\circledR}$ ). These polymers, when forming nanometric systems, may be used in a broad range of targeted drug delivery systems, with a tendency for matrix dissolution in the body (Niranjan Patra et al., 2017). The release mechanism of an entrapped drug can be based on matrix bioerosion, diffusion or the combination of both. It depends on the chemical nature of the polymer (Bal, Sengupta, Murthy, 2013; Gültekin, Değim, 2013). 
In this paper, we have advanced novel Eudragit ${ }^{\mathbb{}}$ based nanoparticles for sustained release of SVT. Despite the extensive research regarding the development of nanoparticles for enhancing the oral bioavailability of low solubility drugs, the use of Eudragit ${ }^{\circledR}$ polymers for developing SVT nanoparticles have not been reported so far. This reinforces the innovative degree of our drug delivery systems. The Eudragit ${ }^{\circledR}$ FS30D (EDGFS) and Eudragit ${ }^{\circledR}$ NE30D (EDGNE) were selected as nanoparticle-forming polymers. The selection of these polymers drew on the possibility of semipermeable matrix formation (EDGNE) and $\mathrm{pH}$-dependent dissolution (EDGFS) as a way to obtain controlled release systems for SVT (Shah et al., 2017; Naiserová et al., 2018). The Eudragit ${ }^{\circledR}$ NE30D nanoparticles (Nano EDGNE+SVT) stand out as a feasible strategy for controlling the release rate of SVT and may be useful for enhancing the bioavailability of such drug.

\section{MATERIAL AND METHODS}

SVT was purchased from Galena Química e Farmacêutica Ltda (Campinas, SP, Brazil); EDG NE30D (EDGNE) or poly-ethylacrylate-co-methylmethacrylate (2:1) and EDG FS30D (EDGFS) or poly-methylacrylateco-methylmethacrylate-co-methacrylic acid (7:3:1) were purchased from Almapal S/A (São Paulo, SP, Brazil); and hydrophilic non-ionic surfactant Polaxamer ${ }^{\circledR} 407$ (P407) was purchased from Embrafarma Produtos Químicos e Farmacêuticos Ltda. (São Paulo, SP, Brazil). All other chemicals were analytical reagent grade.

\section{Preparation of the nanoparticles}

For the preparation of the nanoparticles, the nanoprecipitation technique described elsewhere was used (Fessi et al., 1989). Briefly, $0.100 \mathrm{~g}$ of P407 was weighed and then dissolved in $20 \mathrm{~mL}$ distilled water. Separately, $0.100 \mathrm{~g}$ of polymer (EDGFS or EDGNE) and $0.05 \mathrm{~g}$ of SVT were weighed and then dissolved in 10 $\mathrm{mL}$ of acetone. The compounds were homogenized until a clear organic solution was obtained. Afterwards, the organic solution was poured into the aqueous solution and agitated by magnetic stirring $(120 \mathrm{rpm})$, with the aid of a glass syringe barrel. The suspension formed was evaporated under vacuum using a rotary evaporator (Rotavapor $^{\circledR}$ R-111, Büchi Laboratory Equipment, Switzerland) to remove acetone and obtain an aqueous suspension of nanoparticles. The product remained frozen $\left(-20{ }^{\circ} \mathrm{C}\right)$ until lyophilization. In parallel, the nanoparticle preparation procedure was performed without SVT, which resulted in the formulations used as negative control.

\section{Determination of nanoparticles size distribution, polydispersity index (PDI) and Zeta potential (ZP)}

Dynamic light scattering (DLS) measurements were performed on a Malvern ${ }^{\mathbb{B}}$ Instruments Zetasizer ${ }^{\mathbb{B}}$ ZS Nano Series particle analyzer (Malvern, UK) in which the samples were subjected to a He-Ne laser beam of $632.4 \mathrm{~nm}$ wavelength. In all experiments, ultrapure water obtained from a MilliQ ${ }^{\circledR}$ system (Millipore $^{\circledR}$, Bedford, MA, USA) was used for preparing the samples. The experiments were performed in triplicate. The obtained data were analyzed with Zetasizer software v6.20.

\section{Determination of SVT content}

The quantification of SVT was performed by ultraviolet (UV) absorption spectroscopy using a UVVIS spectrophotometer $(190-1100 \mathrm{~nm})$ model 1600 UV (NOVA Instruments, São Paulo, SP, Brazil). The selected wavelength was $238 \mathrm{~nm}$ (Yu et al., 2017).

The entrapment efficiency of simvastatin was determined by direct method (Zhang et al., 2017). Briefly, the different formulations were determined by centrifugation of the samples at $14,000 \mathrm{~g}$, at $15^{\circ} \mathrm{C}$, for $30 \mathrm{~min}$. The supernatant was filtered and the amount of free simvastatin determined. All measurements were performed in triplicate. The Entrapment Efficiency (EE, $\%)$ was calculated according to the following equation:

\section{Physicochemical characterization by absorption spectroscopy in the infrared region (FT-IR)}

By means of absorption spectroscopy in the infrared region (4000-650 $\mathrm{cm}^{-1}$ ), the Spectrum One ATR (Perkin Elmer, Massachusetts, IL, USA) equipment was used to analyze samples of SVT, polymer (EDGFS or EDGNE), nanoparticles formed only by polymer (Nano EDGFS or Nano EDGNE), and nanoparticles combining polymer EDG and SVT (Nano EDGFS+SVT or Nano EDGNE+SVT). The resulting spectra are the average of 32 scans carried out at a resolution of $4 \mathrm{~cm}^{-1}$. 


\section{Differential Scanning Calorimetry (DSC)}

DSC experiments were simultaneously performed in a Thermogravimetric Analyzer (TGA) SDT-Q600 (TA Instruments, New Castle, DE, USA) under nitrogen atmosphere, at an approximate flow of $50 \mathrm{~mL} \cdot \mathrm{min}^{-1}$. The samples were weighed in alumina crucibles and heated from 25 to $250{ }^{\circ} \mathrm{C}$ at a heating rate of $10^{\circ} \mathrm{C} \cdot \mathrm{min}^{-1}$. The obtained data were analyzed with TA Instruments Universal Analysis 2000 software and were later plotted using Origin ${ }^{\circledR}$ Pro 8.0 software package (OriginLab Corporation, Northampton, MA, USA).

\section{In vitro release of SVT from Eudragit ${ }^{\circledR}$ - based nanoparticles}

The in vitro release assay of SVT was adapted from the pharmacopoeial monograph method described for dissolution test for SVT tablets (Marinho et al., 2011). The dissolution medium used was a phosphate buffer (0.05 mol. $\left.\mathrm{L}^{-1}\right)$ of $\mathrm{pH}$ 7.0, containing sodium lauryl sulfate $\left(0.5 \% \mathrm{w} \cdot \mathrm{v}^{-1}\right)$.

In order to attain sink conditions, $5 \mathrm{mg}$ of pure SVT or samples of nanoparticles containing $5 \mathrm{mg}$ SVT (equivalent to $25 \mathrm{mg}$ of Nano EDGNE+SVT and $33 \mathrm{mg}$ of Nano EDGFS+SVT) were dispersed into the dissolution medium $(900 \mathrm{~mL})$, using the USP type II dissolution apparatus (paddles), at a stirring speed of $50 \mathrm{rpm}$ and $37{ }^{\circ} \mathrm{C} \pm 1{ }^{\circ} \mathrm{C}$ for 5 hours. At predetermined time-points (i.e., 10, 20, 30, 45, 60, 120, 180 and $300 \mathrm{~min}$ ), $5 \mathrm{~mL}$ samples were collected, filtered through a $0.22 \mu \mathrm{m}$ filter (Millipore $^{\circledR}$, Bedford, MA, USA), and then analyzed by absorption spectrophotometry in the ultraviolet region at $238 \mathrm{~nm}$. There was no need for dilution prior to analysis. An equivalent amount of dissolution medium was placed in the dissolutor after each sample withdrawal. The experiments were performed in at least three replicates. Several mathematical models were evaluated in order to predict the drug release kinetics from the nanoparticles. Drug release mechanism was determined by means the diffusional exponent (n) from the "Peppas equation" (Siepmann, Peppas, 2011).

In order to compare the dissolution profiles of the pure drug (SVT), Nano EDGNE+SVT and Nano EDGFS + SVT, the mean drug release amounts $(n=3)$ vs. time were plotted. Furthermore, the Similarity Factor (F2), was calculated as follows (Brasil, 2010):

$$
F 2=50 \times \log \left\{\left[1+\left(\frac{1}{n}\right) \sum_{t=1}^{n}(R t-T t)^{2}\right]^{-0,5} \times 100\right\}
$$

$\mathrm{n}$, is the number of samples withdrawn; Rt, the released amount of drug at $t$ time, obtained for the SVT; Tt, the released amount of drug at $t$ time, obtained for the formulation in test.

\section{Statistical analysis}

The data are presented as the mean \pm standard deviation (SD). The average of two populations was compared by the Student's t-test. The data of three or more populations were compared by analysis of variance (ANOVA) with a subsequent parametric Tukey test. In all of these analyses, $p$ values $<0.05$ at $95 \%$ confidence interval were considered significantly different.

GraphPad Instat 5.0 software (GraphPad Software Inc., CA, USA) was used for data analysis.

\section{RESULTS AND DISCUSSION}

The method selected for preparing the polymeric nanoparticles i.e., nanoprecipitation, has been widely used by both the academic and industrial communities for the development of pharmaceutical and agricultural products as innovative and eco-friendly solutions. The main reasons for preferring the nanoprecipitation technique over the others used in the preparation of polymeric nanoparticles are the easiness and promptness of obtainment, good reproducibility, low organic solvent consumption and exposure for the manufacturer, affordability and easiness of scaling-up (Rivas et al., 2017).

By this method, nanoparticles were formed spontaneously when the organic phase containing one of the polymers (EDGFS or EDGNE) plus SVT dissolved in acetone is poured into the aqueous solution containing surfactant P407, agitated by magnetic stirring, thus resulting in a milky-looking suspension. By mixing the organic with the aqueous solution, a spontaneous dispersion of the organic phase has taken place, forming opalescence systems due to miscibility of both solvents. This process is followed by removal of the organic solvent under reduced pressure to obtain nanoparticles suspended in water (Crucho, Barros, 2017).

Agitation of the system is the key step for obtaining the nanoparticles and must be performed before solvent diffusion and polymer precipitation; hence, droplets of colloidal dimensions can be obtained. Non-ionic 
surfactant P407 is also important because it stabilizes and keeps the system dispersed (Sharma, Madan, Lin, 2016).

\section{The size distribution, $\mathrm{PI}$ and ZP of the polymeric nanoparticle}

Nanoparticles are solid, colloidal particles with size range within $10 \mathrm{~nm}$ to $1000 \mathrm{~nm}$; however, for nanomedical application, the preferential size is less than $200 \mathrm{~nm}$ (Biswas et al., 2014; Rizvi, Saleh, 2018). However, the Zeta potential of these nanoparticles can be positive or negative, depending on the nature of the polymer, drug or material used to modify the surface charge of the system (Reisch et al., 2015). Zeta potential measurements are used to analyze the colloidal stability and the surface charge of nanoparticles (Prabha, Raj, 2016). Table I displays the mean diameter, Polydispersity Index (PDI) and the Zeta Potential (ZP) of the nanoparticles prepared.

It is possible to assert that the selected method produced nanoparticles of diameter less than $200 \mathrm{~nm}$. Noteworthy, it have been described that polymeric nanoparticles with diameter less than $200 \mathrm{~nm}$ present enhanced uptake by Caco-2 and Caco-2/HT-29 cells (Banerjee et al., 2016), and also would be able to cross the hepatocyte membrane and enhance liver retention of SVT.

EDGNE and EDGNE+SVT nanoparticles showed mean sizes with no statistically significant difference $(p$ $>0.05$ ), which suggests that SVT did not interfere in or modify the dimensional structure of the nanoparticles.
As for the mean size of EDGFS+SVT nanoparticles, a significant diameter increase $(\mathrm{p}<0.001)$ was observed when compared to that of EDGFS nanoparticles. In this case, the drug was able to modify the dimensional structure of EDGFS nanoparticles. Some researchers have pointed out that the viscosity of the dispersed phase can be related to the size distribution, where, often increasing the viscosity of the organic phase, the mean size also increases (Pongpaibul, Whitworth, 1986; Chong-Kook, Mi-Jung, Kyoung-Hee, 1994).

Polydispersity index (PDI) characterizes the disperse systems with respect to the deviation from the average size. The PDI values observed for the formulations were lower than 0.26. Lower PDI indicated good dispersibility, a prerequisite of good stability (Cheng, Sun, Gong, 2018; Danaei et al., 2018) indicating that the nanoparticles were homogenous and had narrow size distribution. The qualitative-quantitative composition and method of preparation of the nanoparticles are determinants of the mean diameter and the polydispersity of the particles (Sharma, Madan, Lin, 2016).

With respect to the ZP of each system, it is known that this value is a result of the combination of the materials used, where the drug and the polymers are the main components in the formulations, capable of influencing the Zeta potential. A relatively high Zeta potential value is important to maintain the physicochemical stability of the colloidal suspension, since large repulsive forces tend to avoid aggregation due to occasional collisions of adjacent nanoparticles (Vinayagam, Varadavenkatesan, Selvaraj, 2017)

TABLE I - Mean diameter and Zeta potential of the Eudragit ${ }^{\circledR}$ nanoparticles unloaded and loaded with SVT

\begin{tabular}{lcccc}
\hline & $\begin{array}{c}\text { Nano } \\
\text { EDGFS }\end{array}$ & $\begin{array}{c}\text { Nano } \\
\text { EDGNE }\end{array}$ & Nano EDGFS+SVT & Nano EDGNE+SVT \\
\hline Diameter $(\mathrm{nm})$ & $70.5 \pm 2.44$ & $110.9 \pm 2.04^{\mathrm{a}}$ & $148.8 \pm 20.8$ & $105.0 \pm 12.35^{\mathrm{a}}$ \\
Zeta Potential $(\mu \mathrm{v})$ & $-15.4 \pm 0.42^{\mathrm{ab}}$ & $-14.1 \pm 0.65^{\mathrm{ac}}$ & $-23.5 \pm 4.22$ & $-15.1 \pm 0.89^{\mathrm{bc}}$ \\
Polydispersity Index $(-)$ & $0.083 \pm 0.023$ & $0.225 \pm 0.014^{\mathrm{ab}}$ & $0.256 \pm 0.007^{\mathrm{ac}}$ & $0.245 \pm 0.043^{\mathrm{bc}}$ \\
\hline
\end{tabular}

Mean \pm standard deviation (SD); $\mathrm{n} \geq 3$; unloaded Eudragit ${ }^{\circledR}$ FS30D nanoparticles (Nano EDGFS); unloaded Eudragit ${ }^{\circledR}$ NE30D nanoparticles (Nano EDGNE); simvastatin-loaded Eudragit ${ }^{\circledR}$ FS30D nanoparticles (Nano EDGFS+SVT); simvastatin-loaded Eudragit ${ }^{\circledR}$ NE30D nanoparticles (Nano EDGNE+SVT). The values were analyzed by one-way analysis of variance (ANOVA) and the post hoc Tukey's test, with significance set at $\mathrm{p}<0.05$. No significant differences were observed if mean is quoted with the same letter. 
This study verified that all nanoparticles showed a negative Zeta potential, which may be likely attributed to the presence of ionized carboxyls in EDGFS or due to non-esterified carboxyls still remaining in EDGNE (Figure 2). With the presence of SVT, there was a significant variation of the Zeta potential only for EDGFS+SVT nanoparticles, suggesting that the drug was able to change the surface charge of the nanosphere.

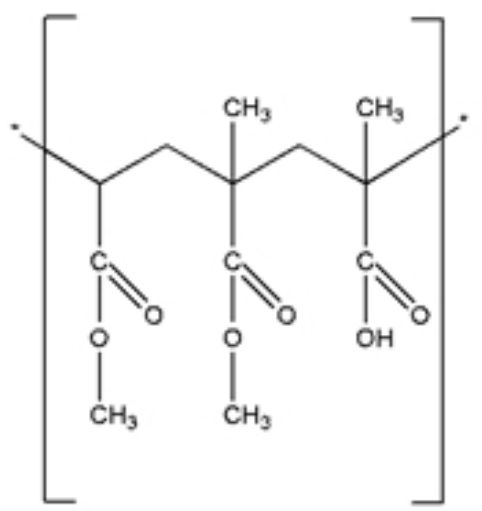

(A)

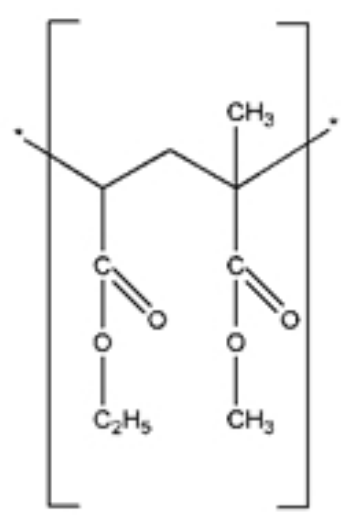

(B)
FIGURE 2 - Monomer of Eudragit ${ }^{\circledR}$ FS30D (A) and Eudragit ${ }^{\circledR}$ NE30D (B).

\section{Entrapment efficiency of SVT}

The entrapment efficiency of SVT in the nanoparticles was $76.4 \%( \pm 3.8)$ for EDGFS + SVT and $103.2 \%( \pm 2.2)$ for EDGNE+SVT $(\mathrm{p}<0.05)$. In both cases, the entrapment efficiency was high due to the low solubility of SVT in the aqueous medium. The amount of drug encapsulated in a nanoparticle may be correlated primarily to their affinity with the organic or aqueous phase during the preparation process. The $\mathrm{O} / \mathrm{W}$ partition coefficient, which is 4.68 for SVT, evinces a strong tendency to maintain the drug in the organic phase of the system. In addition, the greater the affinity of the drug with the polymer, the greater proportions thereof will be associated with the nanoparticles and the higher the entrapment efficiency (Yu et al., 2017).

\section{Characterization by FT-IR}

Figures 3 and 4 show the absorption spectra in the infrared region of SVT, polymers (EDGFS or EDGNE), nanoparticles formed only by a polymer (EDGFS or
EDGNE nanoparticles), and nanoparticles combining a polymer and SVT (EDGFS+SVT or EDGNE+SVT nanoparticles). Moreover, the Table II displays the assignments of the main bands regarding the absorption spectra in the infrared of SVT.

The interpretation of the respective infrared spectra, for the systems prepared either with EDGFS or EDGNE, demonstrated that in EDGFS+SVT nanoparticles (Figure 3) the band is not perceived at $3546 \mathrm{~cm}^{-1}(\mathrm{v}(\mathrm{O}-\mathrm{H}))$, while in EDGNE+SVT nanoparticles (Figure 4) the band can be seen at $3548 \mathrm{~cm}^{-1}$. This band can be assigned specifically to SVT, indicating partial presence of the drug in the crystalline state (Cejka et al., 2003) on the surface of the nanoparticle EDGNE+SVT.

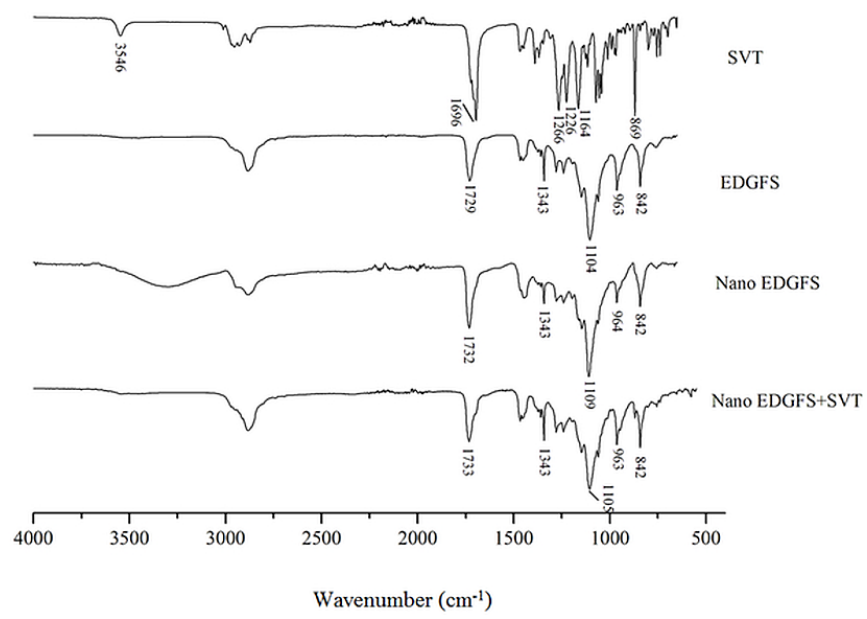

FIGURE 3 - Comparison of absorption spectra in the infrared region for simvastatin (SVT), Eudragit ${ }^{\circledR}$ FS30D (EDGFS), unloaded Eudragit ${ }^{\circledR}$ FS30D nanoparticles (Nano EDGFS) and simvastatin-loaded Eudragit ${ }^{\circledR}$ FS30D nanoparticles; (Nano EDGFS+SVT).

Another band that indicates the presence of SVT on the surface of EDGNE+SVT nanoparticles is $1697 \mathrm{~cm}^{-1}(\mathrm{v}(\mathrm{C}=\mathrm{O}))$, assigned to lactone carbonyl stretching of SVT so that EDGNE+SVT nanoparticles contain a significant amount of SVT on their surface. Conversely, no band assigned to SVT was observed in the spectrum of EDGFS+SVT nanoparticles. This indicates that SVT may be somewhat more entrapped and less exposed on the surface of nanoparticles with the use of EDGFS. 


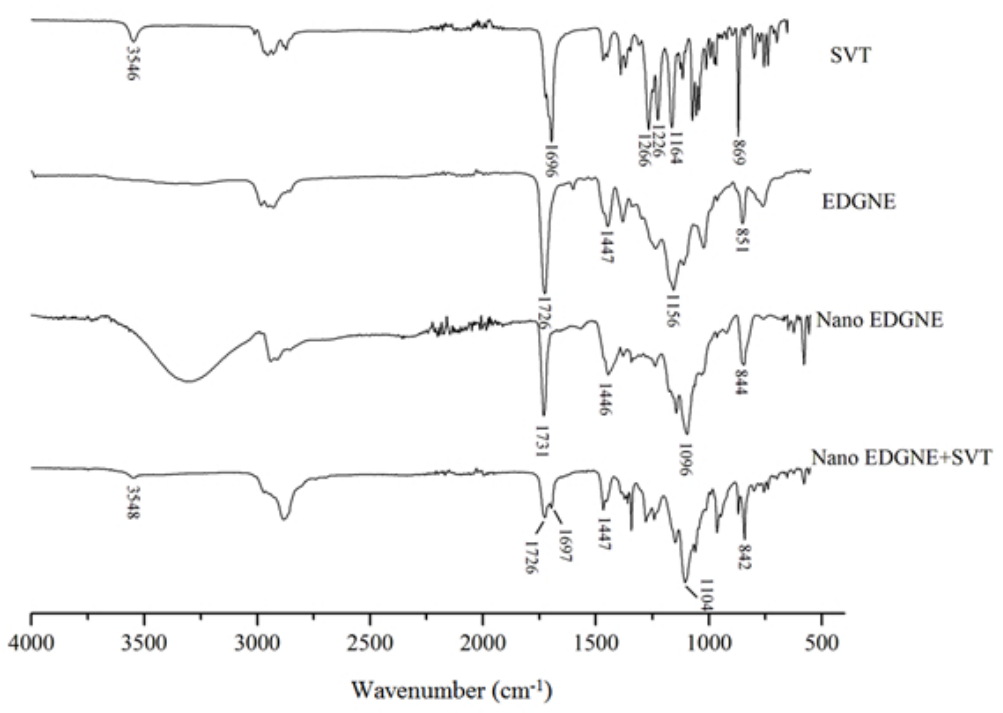

FIGURE 4 - Comparison of absorption spectra in the infrared region for simvastatin (SVT), Eudragit ${ }^{\circledR}$ NE30D (EDGNE), unloaded Eudragit ${ }^{\circledR}$ NE30D nanoparticles (Nano EDGNE) and simvastatin-loaded Eudragit ${ }^{\circledR}$ NE30D nanoparticles (Nano EDGNE+SVT).

TABLE II - Assignments for the main bands in the SVT infrared spectrum of simvastatin-loaded polymeric nanoparticles

\begin{tabular}{lc} 
Wavenumber $\left(\mathbf{c m}^{-1}\right)$ & Assignments* \\
\hline 3547 & Alcohol O-H Stretch \\
1696 & Ester C=O Stretch (lactone - C6") \\
1266 & Ester C-O-C asymmetric bend \\
1164 & Ester C-O-C asymmetric bend \\
869 & Trisubstituted alkene C-H bend \\
\hline
\end{tabular}

*Silverstein, Webster, Kiemle, 2006.

\section{Thermal behavior as determined by DSC}

Another technique used herein to characterize the nanoparticles was Differential Scanning Calorimetry (DSC). The DSC profiles of the samples are shown in Figures $5 \mathrm{a}$ and $5 \mathrm{~b}$. The fusion of SVT was identified at $141{ }^{\circ} \mathrm{C}$. The disappearance of this fusion peak would be useful to track the inclusion of SVT in the nanosphere and would also indicate the possible amorphous state of the material. This is desired because the amorphous condition should contribute to the dissolution rate of the drug. The endothermic fusion of SVT in EDGFS+SVT (Figure 5a) and EDGNE+SVT (Figure 5b) nanoparticles was not identified, which indicates entrapment of the drug and amorph behavior of the nanoparticulate system.

The analysis of the thermograms also allowed observing the glass transition of the polymers used to prepare the nanoparticles. EDG has been described as having a glass transition temperature of approximately $54{ }^{\circ} \mathrm{C}$ (Graeser et al., 2008). 


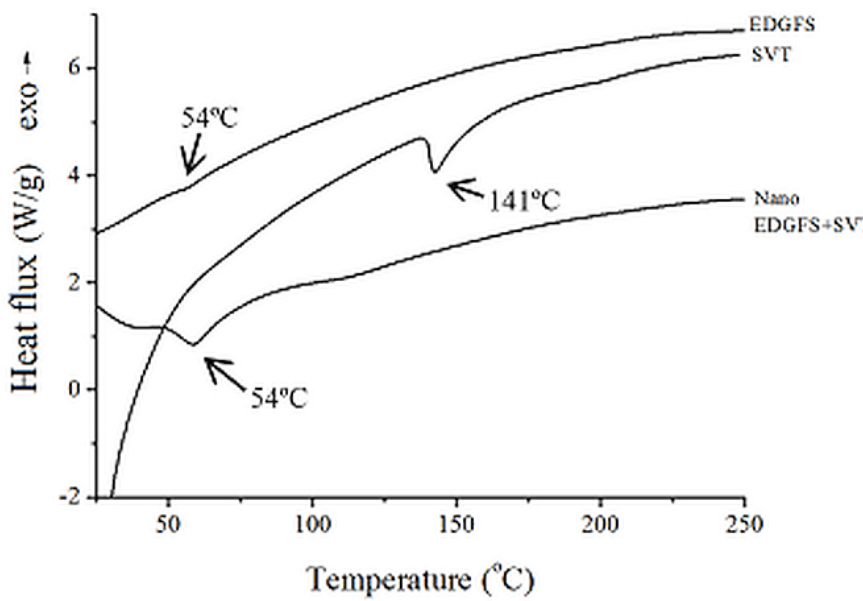

(a)

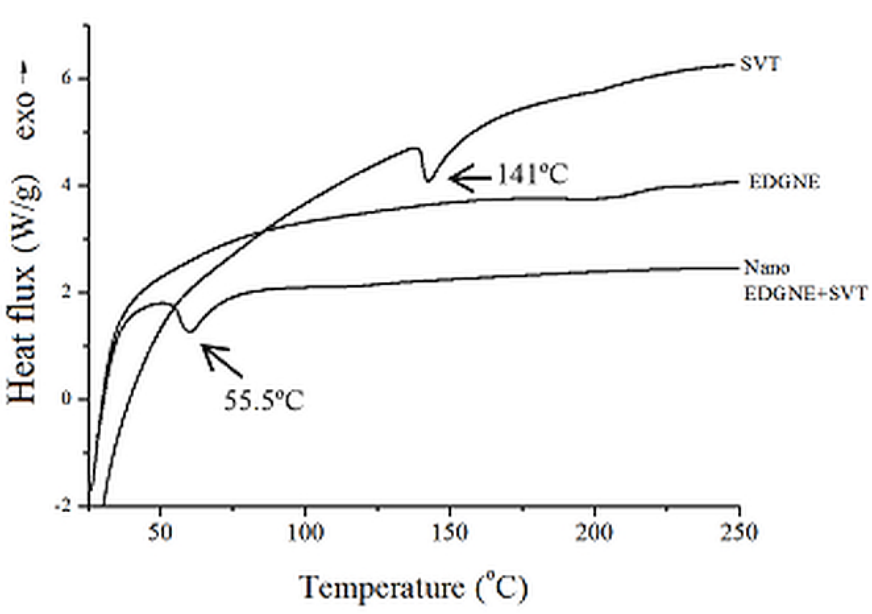

(b)

FIGURE 5 - DSC curves for simvastatin (SVT), Eudragit ${ }^{\circledR}$ FS30D (EDGFS), simvastatin-loaded Eudragit ${ }^{\circledR}$ FS30D nanoparticles (Nano EDGFS+SVT) (a), Eudragit ${ }^{\circledR}$ NE30D (EDGNE) and simvastatin-loaded Eudragit ${ }^{\circledR}$ NE30D nanoparticles (Nano EDGNE+SVT) (b), obtained under a dynamic atmosphere of $\mathrm{N}_{2}$, flow rate of $50 \mathrm{~mL} \mathrm{~min}{ }^{-1}$ and heating rate of $10{ }^{\circ} \mathrm{C} \mathrm{min}{ }^{-1}$.

\section{In vitro release testing}

Drug release from nanoparticles is an important step in the development of effective formulations (Gültekin, Değim, 2013). In vitro drug release has been regarded as a parameter to assess formulation safety and efficacy, being used to reflect the in vivo behavior
(Souza, 2014). In vitro testing helps to select the most appropriate systems for a future in vivo release testing. The profiles of SVT release from the nanoparticles prepared with polymers EDGFS and EDGNE are shown in Figure 6, which also displays the release of pure SVT for comparison.

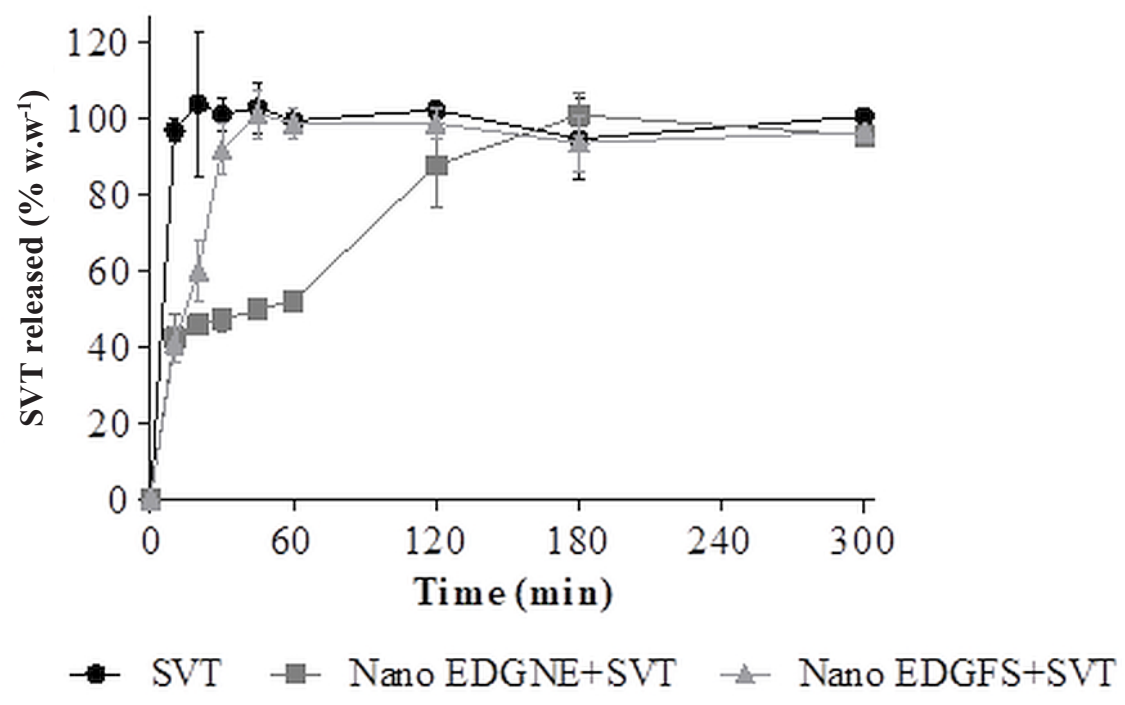

FIGURE 6 - Release profile of nonencapsulated simvastatin (SVT) and SVT incorporated into Nano EDGFS+SVT and Nano EDGNE+SVT. USP type II dissolution apparatus (paddles), $900 \mathrm{~mL}$ of phosphate buffer $\left(0.05\right.$ mol. $\left.\mathrm{L}^{-1}\right) \mathrm{pH}$ 7.0, plus sodium lauryl sulfate $\left(0.5 \% \mathrm{w} \cdot \mathrm{v}^{-1}\right), 37^{\circ} \mathrm{C} \pm 1^{\circ} \mathrm{C}, 50 \mathrm{rpm}(\mathrm{n}=3)$. 
The analysis of the profiles demonstrated that non-encapsulated SVT (pure drug), used as a positive control for dissolution, reaches a $100 \%$ dissolution level 10 minutes after test start. Such a rapid dissolution of SVT was only possible because of the sodium lauryl sulfate surfactant present in the dissolution medium. We have calculated the F2 values to measure the similarity between the dissolution profiles achieved for SVT and Nano EDGFS+SVT and Nano EDGNE+SVT. The obtained results were: 17.1 when comparing SVT with Nano EDGNE+SVT; 28.3 when comparing SVT with Nano EDGFS+SVT; and 24.8 when comparing Nano EDGNE+SVT with Nano EDGFS+SVT. Once all these values were lower than 50, from these findings it is possible to assert that the dissolution assay proposed herein was remarkably discriminative, i.e., the experimental conditions did not affect the SVT dissolution rather the pharmaceutical technology that have been used for controlling its release.

The release of SVT from EDGFS+SVT nanoparticles, however, occurs almost completely at approximately 50 minutes after test start. One of the reasons for this behavior is the $\mathrm{pH}$ used in the dissolution medium, as EDGFS is insoluble at a $\mathrm{pH}$ less than 7.0, but is soluble at a $\mathrm{pH}$ greater than 7.0 (Wagdare et al., 2011). Knowing that the dissolution medium used benefits EDGFS dispersion, it can be stated that the SVT release was rapid because of this chemical characteristic of the polymer (Niranjan Patra et al., 2017).

In the case of EDGNE+SVT nanoparticles, we have observed a bimodal release profile. A rapid release $( \pm$ $45 \%$ ) - or "burst" - of SVT was observed within the first 10 minutes of test, which supports the theory that the drug is present on the surface of the nanoparticle. Subsequently, between the time of 10 and 180 minutes, the SVT release rate lowers and can be described as sustained.

Dissolution profile analysis allows determining the drug release kinetics from the nanoparticles (Abioye, Armitage, Kola-Mustapha, 2016). Among the many mathematical models available, seven different models were empirically selected for the present study. The mathematical models used and the corresponding determination coefficients $\left(\mathrm{r}^{2}\right)$ are shown in Table III.

Knowing that the greater the $\mathrm{r}^{2}$ value the more explanatory the model, that is, the better it fits the sample, the zero-order and Baker-Lonsdale models are, respectively, the ones that best fit and justify the mechanisms of SVT release from the nanoparticles produced with polymers EDGFS and EDGNE.
TABLE III - Mathematical models and their determination coefficients $\left(\mathrm{r}^{2}\right)$ used to characterize release kinetics of simvastatin from Eudragit ${ }^{\circledR}$ polymeric nanoparticles

\section{Determination Coefficient $\left(\mathbf{r}^{2}\right)$}

\begin{tabular}{lcc} 
Kinetic Model & Nano EDGFS+SVT & Nano EDGNE+SVT \\
Baker-Lonsdale & 0.9803 & $\mathbf{0 . 9 8 9 4}$ \\
Higuchi & 0.9837 & 0.9442 \\
Hixson-Crowell & 0.9639 & 0.9791 \\
Zero Order & $\mathbf{0 . 9 9 7 3}$ & 0.9791 \\
Peppas & 0.9900 & 0.9863 \\
First Order & 0.9365 & 0.9856 \\
Weibull & 0.9900 & 0.9863 \\
\hline
\end{tabular}

Bold values represent the most significant models; simvastatinloaded Eudragit ${ }^{\circledR}$ FS30D nanoparticles (Nano EDGFS+SVT);

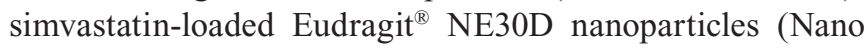
EDGNE+SVT); $\mathrm{n}=3$.

EDGFS+SVT nanoparticles achieve zero-order release kinetics, which is characterized by a continuous release of the same amount of drug per unit time. Matrix systems containing poorly soluble drugs are typically characterized by release kinetics of this nature (Zhang et al., 2017). In turn, EDGNE+SVT nanoparticles achieve SVT release kinetics as per the Baker-Lonsdale model, usually used to describe controlled drug release from spherical matrices (Scioli Montoto et al., 2018).

Regarding the drug release mechanism, based on the power law proposed that represents the "Peppas equation" (Siepmann, Peppas, 2011) it was possible to calculate the diffusional exponent (n) for such spherical drug delivery systems. The $\mathrm{n}$ value for EDGFS+SVT nanoparticles was 0.11 , thus lower than 0.43 which means that the SVT is released by Fickian diffusion. On the other hand, the $\mathrm{n}$ value for $\mathrm{EDGNE}+\mathrm{SVT}$ nanoparticles was 0.51 i.e., $0.43<\mathrm{n}<0.85$, therefore the release mechanism is an anomalous transport, which is and equilibrium between Fickian and Case II (polymer relaxation controlled release).

Eudragit $^{\circledR}$ based nanoparticles have been used to overcome the low solubility and enhance the bioavailability of several drugs e.g., efavirenz (Hari et 
al., 2016), atazanavir (Shing, Pai, 2016) and silymarin (Younis, Shaheen, Abdallah, 2016; El-Nahas et al., 2017). In these cases, remarkable increase in drug concentration in serum and major organs, including liver compared to the free drugs have been reported. Additionally, Abdel-Wahhab et al. (2017) have ratified the low toxicity of Eudragit ${ }^{\circledR}$ polymers nanoparticles in an animal model. Together with their known biocompatibility, Eudragit ${ }^{\mathbb{B}}$ nanoparticles are rapidly extracted from the systemic circulation by the mononuclear phagocytic system and are able to accumulate in the liver (Rolland et al., 1989), thus suggesting that we can reach a liver-targeted delivery of our nanoparticles. Further experimental work need to be performed in order to endorse the liver deposition of Eudragit ${ }^{\circledR}$ NE30D nanoparticles.

These features may be useful for increasing the half-life and, consequently, the bioavailability of SVT in the liver. Moreover, the Eudragit ${ }^{\circledR}$ NE30D nanoparticles have prospect to be used for exploring alternative administration routes e.g., mucosal and transdermal, aiming at to achieve a liver-targeted drug delivery, that will also be the aim of further investigation of our research group.

\section{CONCLUSIONS}

The nanotechnology was herein used envisioning decreasing crystallinity, altering solubility and controlling the velocity and extent of SVT availability. By mean the FT-IR and DSC analysis we have noticed the amorphyzation of the drug. This usually lead to an increase in drug solubility and availability in the desired release site, which is intended to be controlled by the polymer of the engineered delivery matrix. Data arising from the release study indicate that the polymeric nanoparticle obtained with Eudragit ${ }^{\circledR}$ NE30D is capable of releasing the drug in a bimodal controlled manner in vitro i.e., a first phase of "burst release" which is followed by a second phase of prolonged release.

Altogether, these findings and literature reports make it possible to assert that the novel strategy for controlling the drug release based in the use of nanoprecipitation technique and Eudragit ${ }^{\circledR}$ NE30D as matrix polymer could be extended for lipophilic statins with diminished half-life and low bioavailability (e.g, lovastatin) and drugs used in the treatment of hepatic illness such as cirrhosis and cancer.

\section{REFERENCES}

Abdel-Wahhab MA, Joubert O, Khadrawy YA, Safar R, ElNekeety AA, Ronzani C, et al. Preliminary safety assessment of Eudragit ${ }^{\circledR}$ polymers nanoparticles administration in the rat brain. J App Pharm Sci. 2017;7(7):176-185.

Abioye AO, Armitage R, Kola-Mustapha AT. Thermodynamic changes induced by intermolecular interaction between ibuprofen and chitosan: effect on crystal habit, solubility and in vitro release kinetics of ibuprofen. Pharm Res. 2016;33(2):337-57.

Alshora DH, Ibrahim MA, Elzayat E, Almeanazel OT, Alanazi F. Rosuvastatin calcium nanoparticles: Improving bioavailability by formulation and stabilization codesign. PLoS One. 2018;13(7):1-17.

Bal T, Sengupta S, Murthy PN. Formulation and evaluation of carvedilol microcapsules using Eudragit NE30D and sodium alginate. Braz J Pharm Sci. 2013;49(4):889-901.

Basu A, Kunduru KR, Katzhendler J, Domb AJ. Poly $(\alpha-$ hydroxy acid)s and poly( $\alpha$-hydroxy acid-co- $\alpha$-amino acid)s derived from amino acid. Adv Drug Deliv Rev. 2016;107:82-96.

Banerjee A, Qi J, Gogoi R, Wong J, Mitragotri S. Role of nanoparticle size, shape and surface chemistry in oral drug delivery. J Control Release. 2016;238:176-185.

Biswas AK, Islam MR, Choudhury ZS, Mostafa A, Kadir MF. Nanotechnology based approaches in cancer therapeutics. Adv Nat Sci Nanosci Nanotechnol. 2014;5(4):043001.

Brasil. Agência Nacional de Vigilância Sanitária. Ministério da Saúde. Resolução de Diretoria Colegiada (RDC) No 31, de 11 de agosto de 2010 .

Cejka J, Kratochvil B, Cisarova I, Jegorov A. Simvastatin. Acta Crystallogr. C. 2003;59(Part 8):428-430.

Cheng K, Sun S, Gong X. Preparation, characterization, and antiproliferative activities of biotin-decorated docetaxelloaded bovine serum albumin nanoparticles. Braz J Pharm Sci. 2018;54(2):1-11.

Chong-Kook K, Mi-Jung K, Kyoung-Hee O. Preparation and evaluation of sustained release microspheres of terbutaline sulfate. Int J Pharmaceut. 1994;106(3):213-219.

Crucho CIC, Barros MT. Polymeric nanoparticles: A study on the preparation variables and characterization methods. Mater Sci Eng C Mater Biol Appl. 2017;80:771-784. 
Cruz JN, Dal Magro DD, Lima DD, Cruz JGP. Simvastatin treatment reduces the cholesterol content of membrane/ lipid rafts, implicating the N-methyl-D-aspartate receptor in anxiety: a literature review. Braz J Pharm Sci. 2017;53(1): $1-15$.

Cunha e Rocha K, Pereira BMV, Rodrigues AC. An update on efflux and uptake transporters as determinants of statin response. Exp Opin Drug Metab Toxicol. 2018;14(6):613-24.

Danaei M, Dehghankhold M, Ataei S, Hasanzadeh Davarani F, Javanmard R, Dokhani A, et al. Impact of particle size and polydispersity index on the clinical applications of lipidic nanocarrier systems. Pharmaceutics. 2018;10(2):1-17.

El-Nahas AE, Allam AN, Abdelmonsif DA, El-Kamel AH. Silymarin-loaded eudragit nanoparticles: formulation, characterization, and hepatoprotective and toxicity evaluation. AAPS Pharm Sci Tech. 2017;18(8):3076-3086.

Fattahi A, Karimi-Sabet J, Keshavarz A, Golzary A, RafieeTehrani M, Dorkoosh FA. Preparation and characterization of simvastatin nanoparticles using rapid expansion of supercritical solution (RESS) with trifluoromethane. J Supercrit Fluids. 2016;107:469-478.

Fessi H, Puisieux F, Devissaguet JP, Ammoury N, Benita $\mathrm{S}$. Nanocapsule formation by interfacial polymer deposition following solvent displacement. Int $\mathrm{J}$ Pharmaceut. 1989;55(1):R1-R4.

Graeser KA, Strachan CJ, Patterson JE, Gordon KC, Rades T. Physicochemical properties and stability of two differently prepared amorphous forms of simvastatin. Cryst Growth Des. 2008;8(1):128-35.

Gültekin HE, Değim Z. Biodegradable polymeric nanoparticles are effective systems for controlled drug delivery. FABAD J Pharm Sci. 2013;38(2):107-118.

Hari BNV, Narayanan N, Dhevendaran K, Ramyadevi D. Engineered nanoparticles of Efavirenz using methacrylate co-polymer (Eudragit ${ }^{\circledR}$-E100) and its biological effects invivo. Mat Sci Eng C. 2016;67:522-532.

Korani S, Korani M, Bahrami S, Johnston TP, Butler AE, Banach M, Sahebkar A. Application of nanotechnology to improve the therapeutic benefits of statins. Drug Discov Today. 2018;S1359-6446(18)30324-6.

Marinho FDM, Diniz MML, Zanon JCC, Reis IA, Lima AA, Soares CDV. Influência da temperatura em estudos de dissolução de formas farmacêuticas contendo sinvastatina. Rev Bras Farm. 2012;93:38-42
Naiserová M, Kubová K, Vysloužil J, Pavloková S, Vetchý D, Urbanová M, et al. Investigation of dissolution behavior HPMC/Eudragit. AAPS PharmSciTech. 2018;19(2):681-692

Niranjan Patra CH, Priya R, Swain S, Jena GK, Panigrahi KC, Ghose D. Pharmaceutical significance of Eudragit: A review. Future J Pharm Sci. 2017;3:33-45.

Petyaev IM. Improvement of hepatic bioavailability as a new step for the future of statin. Arch Med Sci. 2015;11(2):406-410.

Pongpaibul Y, Whitworth CW. Preparation and in vitro dissolution characteristics of propranolol microcapsules. Int J Pharm. 1986;33(1):243-248.

Prabha G, Raj V. Preparation and characterization of polymer nanocomposites coated magnetic nanoparticles for drug delivery applications. J Magn Magn Mater. 2016;408:26-34.

Reisch A, Runser A, Arntz Y, Mély Y, Klymchenko AS. Charge-controlled nanoprecipitation as a modular approach to ultrasmall polymer nanocarriers: making bright and stable nanoparticles. ACS Nano. 2015;9(5):5104-5116.

Rizvi SAA, Saleh AM. Applications of nanoparticle systems in drug delivery technology. Saudita Pharm J. 2018;26(1):64-70.

Rivas CJM, Tarhini M, Badri W, Miladi K, Greige-Gerges H, Nazari QA, et al. Nanoprecipitation process: From encapsulation to drug delivery. Int J Pharm. 2017;532(1): 66-81.

Rolland A, Collet B, Le Verge R, Toujas L. Blood clearance and organ distribution of intravenously administered polymethacrylic nanoparticles in mice. J Pharm Sci. 1989;78(6):481-484.

Scioli Montoto S, Sbaraglini ML, Talevi A, Couyoupetrou M, Di Ianni M, Pesce GO, et al. Carbamazepine-loaded solid lipid nanoparticles and nanostructured lipid carriers: Physicochemical characterization and in vitro/in vivo evaluation. Colloids Surf B Biointerfaces. 2018;167:73-81.

Shah SU, Socha M, Sejil C, Gibaud S. Spray-dried microparticles of glutathione and S-nitrosoglutathione based on Eudragit. Ann Pharm Fr. 2017;75(2):95-104.

Sharma N, Madan P, Lin S. Effect of process and formulation variables on the preparation of parenteral paclitaxel-loaded biodegradable polymeric nanoparticles: A co-surfactant study. Asian J Pharm. 2016;11(3):404-416.

Shilpi D, Kushwah V, Agrawal AK, Jain S. Improved stability and enhanced oral bioavailability of atorvastatin loaded stearic acid modified gelatin nanoparticles. Pharm Res. 2017;34(7):1505-1516. 
Singh G, Pai RS. Atazanavir-loaded Eudragit RL 100 nanoparticles to improve oral bioavailability: optimization and in-vitro/in-vivo appraisal. Drug Deliv. 2016;23(2):532-539.

Siepmann J, Peppas NA. Higuchi equation: Derivation, applications, use and misuse. Int J Pharm. 2011;418(1):6-12.

Silverstein RM, Webster FX, Kiemle DJ. Identificação espectrométrica de compostos orgânicos. 7.ed. Rio de Janeiro: LTC; 2007. 490 p.

Souza SD. A review of in vitro drug release test methods for nano-sized dosage forms. Adv Pharm. 2014;2014:304757.

Stone NJ, Robinson JG, Lichtenstein AH, Bairey Merz CN, Blum CB, Eckel RH, et al. 2013 ACC/AHA guideline on the treatment of blood cholesterol to reduce atherosclerotic cardiovascular risk in adults: a report of the American College of Cardiology/ American Heart Association Task Force on Practice Guidelines. J Am Coll Cardiol. 2014;63(25):2889-2934.

Taha EI. Bioavailability assessment of hydroxymethylglutaryl coenzyme A reductase inhibitor utilizing pulsatile drug delivery system: a pilot study. Drug Deliv. 2016;23(7):2139-2143.

Thompson PD, Panza G, Zaleski A, Taylor B. Statin-associated side effects. J Am Coll Cardiol. 2016;67(20):2395-2410.
Vinayagam R, Varadavenkatesan T, Selvaraj R. Evaluation of the anticoagulant and catalytic activities of the bridelia retusa fruit extract-functionalized silver nanoparticles. J Cluster Sci. 2017;28(5):2919-2932.

Wagdare NA, Marcelis AT, Boom RM, van Rijn CJ. Porous microcapsule formation with microsieve emulsification. J Colloid Interface Sci. 2011;355(2):453-457.

Younis N, Shaheen MA, Abdallah MH. Silymarin-loaded Eudragit ${ }^{\circledR}-\mathrm{S} 100$ nanoparticles improved the abilityof silymarin to resolve hepatic fibrosis in bile duct ligated rats. Biomed Pharmacother. 2016;81:93-103.

Yu WL, Sun TW, Qi C, Zhao HK, Ding ZY, Zhang ZW, et al. Enhanced osteogenesis and angiogenesis by mesoporous hydroxyapatite microspheres-derived simvastatin sustained release system for superior bone regeneration. Sci Rep. 2017;7(1):1-16.

Zhang Y, Ng W, Feng X, Cao F, Xu H. Lipid vesicular nanocarrier: Quick encapsulation efficiency determination and transcutaneous application. Int $\mathrm{J}$ Pharm. 2017; 516(1):225-230.

Received for publication on $08^{\text {th }}$ May 2018 Accepted for publication on $10^{\text {th }}$ January 2019 